\title{
Wearable Smart Watch Using IOT (Internet of Things)
}

\author{
Dr.T.S.Udhaya Suriya ${ }^{1}$, N.Gokul ${ }^{2}$, L.Sivashankar ${ }^{3}$, P.Sriram $^{4}$, R.Subash ${ }^{5}$ \\ Head of the Department, Bio Medical Department, Adhiyamaan College of Engineering ${ }^{1}$ \\ Student ${ }^{2,3,4,5}$, Bio Medical Department, Adhiyamaan College of Engineering ${ }^{2,3,4,5}$ \\ Email: tsu.suriya@gmail.com ${ }^{1}$,ngokul156gmail.com ${ }^{2}$,sivashankarloganathan@gmail.com ${ }^{3}$, \\ sriramraja@gmail.com ${ }^{4}$,subashdoss@gmail.com ${ }^{5}$
}

\begin{abstract}
Internet of Things (IoT) has attracted lots attention in current years for its potential to relieve the pressure on healthcare structures as a result of an getting older population and a rise in continual illness. Standardization is a key issue restricting progress in this vicinity, and as a consequence this venture proposes a widespread model for application in destiny internet of factors healthcare systems. This survey challenge then presents the modern studies relating to every area of the version, evaluating their strengths, weaknesses, and normal suitability for a wearable IoT healthcare gadget. Demanding situations that healthcare IoT faces inclusive of protection, privateness, wearability and coffee-electricity operation are supplied, and suggestions are made for destiny research instructions.
\end{abstract}

Index Terms- Internet of Things (IoT), Nano Microcontroller, Wearable Sensors.

\section{INTRODUCTION}

The internet of things is the inter-connection of devices, apps, sensors and network connectivity that complements these entities to collect and change facts. The distinguishing feature of net of things inside the healthcare machine is the constant monitoring a affected person through checking diverse parameters and additionally infers a very good result from the records of such steady tracking. Many such devices geared up with medical sensors are present in the ICUs now-a-days. There may be instances where the medical doctor couldn't be alerted in time whilst there may be an emergency, despite of 24 hours of monitoring. Also there might be hurdles in sharing the data and information with the specialist doctors and the concerned family members and relatives. The generation that complements these features is already available but is not accessible and less costly by the general public in developing international locations which include India. Healthcare is a critical part of existence. Sadly, the gradually ageing populace and the associated upward thrust in chronic illness is putting large strain on current healthcare systems, and the demand for sources from sanatorium beds to docs and nurses is extraordinarily high. Clearly, an answer is required to reduce the stress on healthcare systems while continuing to provide terrific care to at-hazard patients.

The internet of things (IoT) has been drastically recognized as a capacity approach to alleviate the pressures on healthcare systems, and has for that reason been the focus of a whole lot present day research. A considerable amount of this research looks at monitoring patients with specific conditions, such as diabetes or Parkinson's disease. Further research looks to serve specific purposes, such as aiding rehabilitation through constant monitoring of a patient's progress. Emergency healthcare has also been identified as a possibility by related works but has not yet been widely researched.

Several related works have formerly surveyed particular regions and technologies associated with IoT healthcare. an in depth survey is provided in with recognition located on commercially to be had solutions, viable programs, and closing issues. Each subject matter is considered one after the other, instead of as part of an overarching gadget. In data mining, storage, and analysis are considered, with little mention of integration of these into a system. Sensor sorts are compared in with some focus placed on communications. However, it is tough to draw a photo of an entire gadget from this paper. Eventually, sensing and huge facts control is taken into consideration, with little regard for the community that will help communications.

This paper therefore makes a unique contribution in that it identifies all key components of a cease-to-quit net of things healthcare machine, and proposes a established model that could be implemented to all IoT-based totally healthcare structures. That is essential as there are nonetheless no known stop-to-stop systems for remote tracking of health inside the literature. This paper in addition offers a comprehensive survey of the today's technology that fall within the proposed version. Focus is placed on sensors for monitoring various health parameters, short- and long-range communications standards, and cloud technologies. Distinguishes itself from the previous predominant survey contributions by thinking about each vital aspect of an IoT-based totally healthcare system each one at a time and as a device.

In addition authentic contribution is made by using setting attention on LPWANs, highlighting their specific suitability to be used in IoT systems. The approaching certified-band standards, consisting of NB-IoT, are in comparison with the competing 


\section{Available online at www.ijrat.org}

unlicensed-band requirements, with precise hobby in suitability for healthcare packages.

\section{LITERATURE SURVEY}

IoT is a new reality that completely changes our daily life. It is also a way to revolutionize present day fitness care by using presenting extra personalized and preventive care ${ }^{[2]}$. There is a high probability that the patient will lose his or her life as a result of excessive fatigue of the heart during sleep at night, especially in Chronic Obstructive Pulmonary Diseases (COPD) and Obstructive Sleep Apnea Syndrome (OSAS) ${ }^{[1]}$. One of these important opportunities is the e-health services that are closely related to all of us. IoT applications in the health sector are increasing day by day. People living in rural areas cannot benefit from preventive health services due to lack of infrastructure ${ }^{[3]}$. Demanding situations that healthcare IoT faces including security, privateness, wear ability, and occasional-strength operation are offered, and suggestions are made for future studies directions ${ }^{[4]}$.

\section{METHODLOGY}

\subsection{Block Diagram}

The Block diagram for the Wearable Smart Watch

Using IoT (Internet of Things) is shown below:

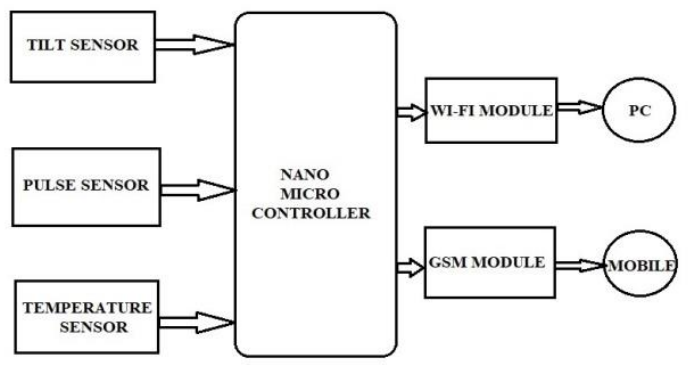

Fig 1 Block Diagram

\subsection{Block Diagram Description}

Nano microcontroller is the central server for the wearable health watch system. It reads the sensed values in the Tilt Sensor, Pulse Sensor and Temperature Sensor, and then the values are displayed in the LCD display. Tilt sensor which senses the body position of the patient's. Pulse sensor senses the pulse of the patient. Lastly, Temperature sensor for sensing the patient's body temperature.Wi-Fi module device will sends the data to the System server in the Hospital. GSM Module is which gives the notification alert to the Mobile Phones of the Patient's neighbour. Buzzer is used to indicate the abnormal values.

And also a $3.7 \mathrm{~V}$ Battery is also connected to the Nano Microcontroller for power supply.

\section{OUTPUT}

Thus the Body Position, Temperature and Pulse of Patient is shown in the output of the model.

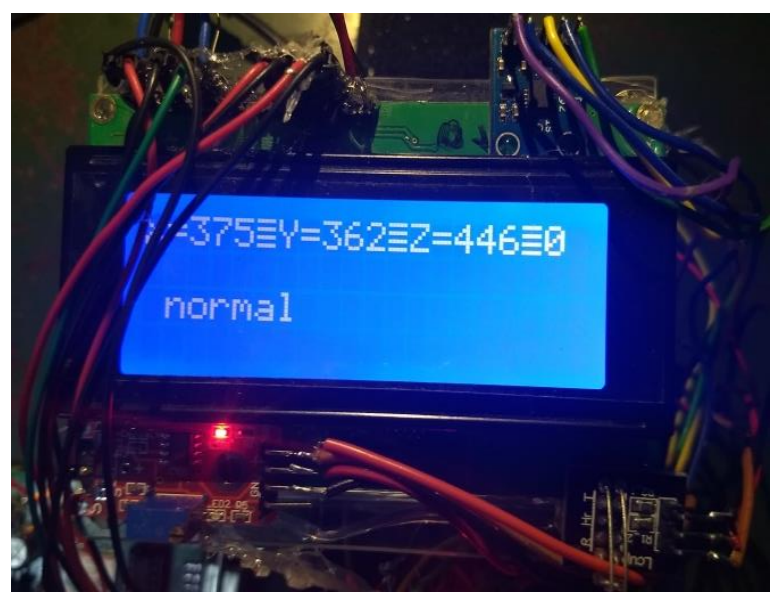

Fig 2 Model Kit of Wearable Smart Watch

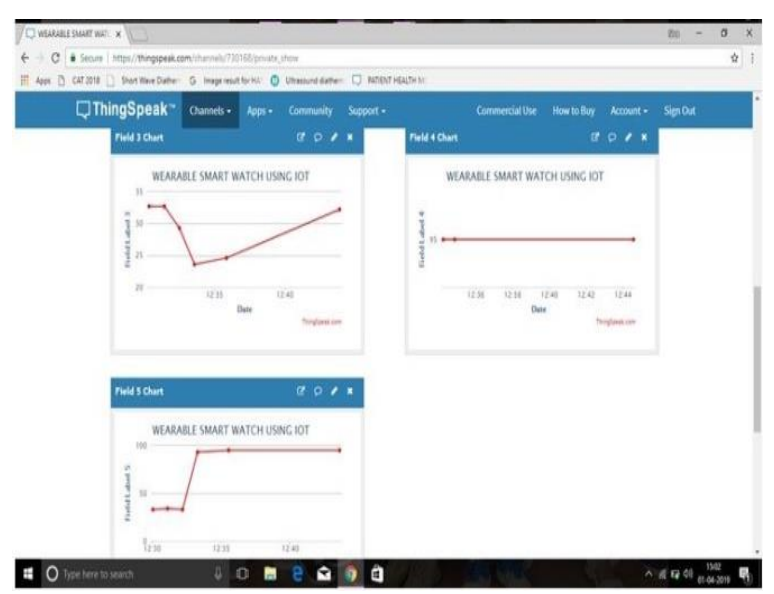

Fig 3 Body Temperature and Humidity of Patient 


\section{$\leftarrow 09786828486$ b. Q :}

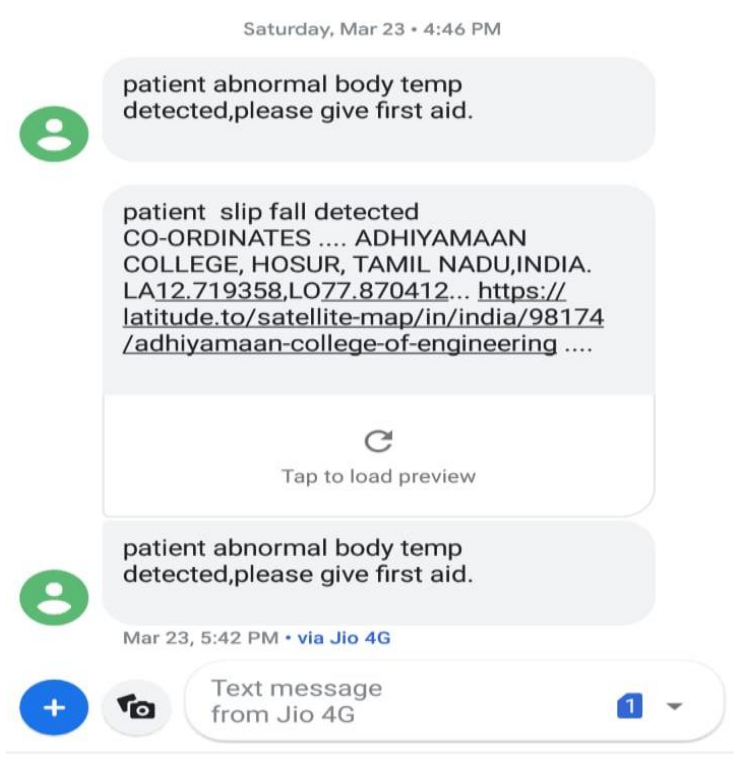

Fig 4 Alert message from the Watch

\section{CONCLUSION}

Increase in the demand of healthcare and biomedical industries leads issues in their efficiency of handling system. Several wearable, non-intrusive sensors were presented and analysed, with particular focus on those monitoring vital signs of patients. In this work, we proposed a unique model for future IoTbased healthcare systems, which can be applied to both general systems and systems that monitor specific conditions. The smart phone integration with healthcare system for monitoring patient's vital health information is made to have significant impact in the healthcare field.

The smart health care reduces the health care cost and also manages the shortage of nursing staff.

\section{FUTURE SCOPE}

Further we extend the existing system to predict if the patient is suffering from any chronic disorder or disease using the various health parameter and various other symptoms that are obtained by the system.

\section{ACKNOWLEDGMENT}

We thankful to Dr. T. S. UDHAYA SURIYA, M.E., Ph.D., Head of the Department of Biomedical Engineering, Adhiyamaan College of Engineering for her valuable guidance and support to complete our project successfully.

\section{REFERENCES}

[1] Agrawal.S, Saha.A, “Advanced IOT based combined remote health monitoring. In Computing and Communication Workshop and Conference", IEEE 8th Annual, pages 602-606, 2014.

[2] Lei.L, Zheng.K, Xiang.W, "Personalized wearable systems for real- time ECG classification and healthcare interoperability: Real- time ECG classification and FHIR interoperability, In Internet Technologies and Applications". International Journal of Computer Networks \& Communications, Volume 2, pages 9-14, 2016.

[3] Thibaud. M, Chi.H, Zhou.W, Piramuthu.S (2015), "Internet of Things (IoT) in high-risk Environment".Decision Support Systems, pages 79-95, 2018.

[4] Wei xiang, Stephanie B.Baker, "Internet of Things for Smart Healthcare: Technologies, Challenges and Opportunities". IEEE, pages 2639, 2017. 